Abstract ID: 83

\title{
Charateristics and reasons for diabetic-defaulters between primary care clinics and diabetic specialist clinic: A prospective cohort study in Kuantan, Malaysia
}

\author{
Fa'iza Abdullah ${ }^{\mathrm{a}}$ | Tin Myo Han ${ }^{\mathrm{b}}$ | Mohd Aznan Md Aris ${ }^{\mathrm{a}}$ | Iskandar Firzada Osman ${ }^{\mathrm{c}}$ \\ ${ }^{a}$ Kulliyyah of Medicine, International Islamic University Malaysia \\ ${ }^{b}$ Kulliyyah of Dentistry, International Islamic University Malaysia \\ ${ }^{c}$ Family Medicine Specialist, Klinik Kesihatan Jaya Gading, Kuantan, Pahang
}

Introduction: Defaulted appointment in diabetic clinics is a great concern as it affects disease controlled and complications. Geographical location, clinic-types and quality of health services provided are known determinant reasons for defaulting. Thus, this study aimed to identify characteristics and reasons for default between diabetic-patients at public-primary-care-clinics (PPCCs) and public-hospital-diabetic-specialist-clinic (DS-OPD). Methods: A prospective one-year-cohort study was conducted among 405 diabetic patients from two PPCCs and DS-OPD in Kuantan (2015-2016). There were 2-point visits (at 6-month and 12-month) assessing follow-up appointments within one year. Defaulter is defined by at least one-time defaulted either at 6-month or 12-month. Regular-attendees were included as control. Type-1-DM-patients, missing-records, known-deceased and transferout cases were excluded. Background socio-demographic data of diabetic-defaulters were collected from DM-records and reasons for defaulting were traced via 3-times-telephone contacts which $51.6 \%$ diabetic-defaulters responded. A stratified cross-analysis was done to compare the prevalence and characteristics between defaulters and regular attendees. Reasons for defaults were analyzed using open-ended-questions analysis method. Results: Prevalence of defaulters was 18\% (73/405); higher prevalence was found in DS-OPD than PPCCs (32.4\% vs 10.3\%). Gender, race, age, education, occupation and the duration of DM were not significantly different between defaulters and regular-attendees at DS-OPD. However, self-employment (25.9\%), housewives (25.9\%), aged less than 45-years (33.3\%) and $\geq 55$ years-old $(44.4 \%)$ were significant defaulters in PPCCs. Significant different of reasons for default found at DS-OPD compare to PPCCs for postponing the date $(54.5 \%$ vs $12.5 \%)$, while refusing treatment/used alternative medicine (18.2\% vs $43.8 \%$ ); and movedout/transferred/referred cases $(27.3 \%$ vs $31.2 \%)$ were more in PPCCs. Conclusions: Distinctive characteristics and diverse reasons for default between DS-OPD and PPCCS among diabetic-patients fortified to set tailored remedial to reduce defaulter-rate in different clinic.

KEYWORDS: Diabetic defaulters, public-primary-care-clinics (PPCCs), public-hospital-diabeticspecialist-clinic (DS-OPD) 